\title{
Comparison of three-dimensional printing guides and double-layer guide plates in accurate bracket placement
}

\author{
Yue Zhang ${ }^{\dagger}$, Chunhao Yang ${ }^{\dagger}$, Yanfeng Li', Dong Xia, Tingting Shi and Changjian Li
}

\begin{abstract}
Background: In the current study, we aimed to evaluate the accuracy of indirect bonding by either threedimensional (3D) printing guides or double-layer guide plates. The results may serve as a clinical reference for bracket placements.

Methods: In total, 140 teeth were collected and arranged into five pairs of full dentition. The marking points were labeled on the buccal/labial surface of the crown in these orthodontic study models. (1) 3D printing guide: A digital profile was generated using an intraoral scanner. Two types of indirect bonding guide, namely the whole denture type and the single tooth type, were designed with the 3Shape TRIOS ${ }^{\circledast}$ Standard intraoral scanner and fabricated using 3D printing technology. (2) Double-layer guide plate: A working model was obtained by replicating the experimental models, and the double-layer guide plate was then made of the inner layer soft film $(1.0 \mathrm{~mm}$ thickness) and the outer layer hard film ( $0.6 \mathrm{~mm}$ or $0.8 \mathrm{~mm}$ thickness). Brackets were transferred from working models to study models by the indirect bonding trays. We measured and analyzed the distance between marking points and bracket placement. Statistical analysis was done using SPSS 20.0 software. The accuracy of indirect bonding between 3D printing guide and double-layer guide plate was compared using paired $t$-test.
\end{abstract}

Results: According to our data, there was a significant difference between the $0.6 \mathrm{~mm}$ group and $0.8 \mathrm{~mm}$ group when the brackets were indirectly adhered using double-layer guide plates $(p=0.036)$. However, no statistical significance in bracket positioning accuracy was revealed between two types of $3 D$ printing guide $(p=0.078)$, as well as between the 3D printing guide group and the $0.6 \mathrm{~mm}$ double-layer guide plate group $(p=0.069)$.

Conclusions: When applying double-layer guide plates for indirect bonding, the $0.6 \mathrm{~mm}$ group is more accurate than the $0.8 \mathrm{~mm}$ group. When utilizing 3D printing guides for indirect bonding, whole denture type is more accessible than single tooth type but with no significant difference in accuracy. The accuracy of indirect bonding is comparable when using 3D printing guides (whole denture type) and double-layer guide plates $(0.6 \mathrm{~mm})$.

Keywords: Indirect bonding, Double-layer guide plate, Three-dimensional printing guide, Accuracy, Bracket placement

\footnotetext{
*Correspondence: 949427779@qq.com

${ }^{\dagger}$ Yue Zhang and Chunhao Yang contributed equally to this work. Department of Stomatology, the Fourth Medical Center, Chinese PLA

General Hospital, No.51 Fucheng Road, Haidian District, Beijing 100048, China
} 


\section{Background}

The positioning and bonding of orthodontic brackets significantly affect the clinical outcome and is the most critical step in orthodontic treatment [1]. Orthodontic brackets can be adhered to the tooth surfaces by direct or indirect bonding. Due to the presence of saliva and some inaccessible tooth positions, direct bonding usually takes longer chair-side time and lacks accuracy [2]. To optimize the accuracy of bracket positioning, indirect bonding technique was proposed by Silverman and Cohen in 1972 and since then became a popular alternative $[1,3]$. It has been shown that errors associated with bracket positioning were minimized in the aspects of height, mesiodistal position, and angulation when using indirect bonding [4]. There are two stages in the indirect bonding procedure, the laboratory stage and the clinical stage [2]. After obtaining patients' orthodontic models, brackets are bonded to the study models in the laboratory stage, and then placed on tooth surfaces integrally with the aid of a customized transfer tray in the clinical stage $[5,6]$. Though the chair-side time is significantly reduced, there are considerable laboratory-associated expenses when indirect bonding is chosen [7].

With the increasing applications of indirect bonding, various designs of transfer trays and novel technologies are implemented in the treatment procedure. In the laboratory stage, the patients' occlusal interrelationship can be duplicated either by impression or digital scanning. The former is a traditional method to generate double-layer guide plates; though with a lower cost, this method typically takes longer laboratory time and is susceptible to human errors. The latter is incorporated with cutting-edge 3D printing technology that provides various advantages, such as precise 3D images, convenience in file storage, and accuracy in image analysis and outcome prediction [5]. Currently, there is limited information on the comparison between traditional indirect bonding and digital indirect bonding in bracket positioning accuracy. To investigate differences in bracket positioning accuracy, we designed different types of transfer trays by traditional impression or 3D printing technology, and then performed a comprehensive evaluation of the clinical efficacy of each design. The data will provide valuable clinical guidance for bracket placements.

\section{Methods}

\section{Fabrication of study models}

We collected 140 teeth with normal crown morphology and no evident defects or restorations at the buccal/labial surfaces, and then sterilized them for the following study. The teeth were arranged into five pairs of complete dentition with mild malocclusion but no torsion/tilting/overlapping. The roots were embedded in denture-base self- curing resin with crowns exposed. The marking points were labeled at the mid of the cervical third and distal end of the incisal third on the study models (Fig. 1).

\section{Production of 3D printing guides and indirect bonding procedure}

Digital models were generated using 3Shape TRIOS ${ }^{\circ}$ Standard intraoral scanner (Fig. 2a). 3Shape OrthoAnanlyzer $^{\text {rux }}$ software was applied to label the marginal gingiva and establish the axis and center of individual crowns, as well as the occlusal plane (Fig. 2b-c). The positioning of the brackets was determined on the digital 3D model (Fig. 2c-f). Finally, guide plates were generated by 3D printing technology (Project 3510 DP) as the whole denture type (Fig. $2 \mathrm{~g}$ ) and single tooth type (Fig. 2h).

Before the indirect bonding procedure, we polished the tooth surfaces, etched buccal/labial surfaces of the crowns for $15 \mathrm{~s}$, and isolated the area with cotton rolls and dried $\mathrm{xx}$ thoroughly. The brackets were positioned in the $3 D$ printing guides (the whole denture type or the single tooth type), and $3 \mathrm{M}$ Unitek Transbond ${ }^{\mathrm{Tm}} \mathrm{XT}$ light-curable adhesives were applied to the base of the brackets (Fig. 3a-b). The $3 D$ printing guides were then placed on the study models, and each border of the brackets was light-cured for $5 \mathrm{~s}$ (Fig. 3c-d). Thereafter, the $3 D$ printing guides were removed to clean excess adhesives around the brackets (Fig. 3e-f).
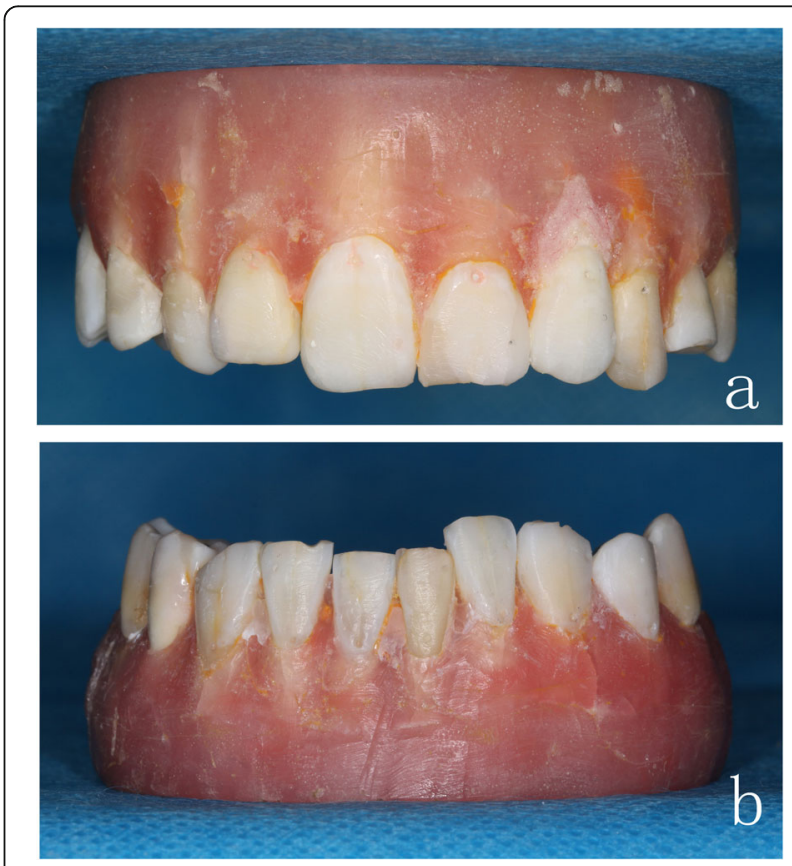

Fig. 1 The study model. a Maxillary model with marking points. b Mandibular model with marking points 


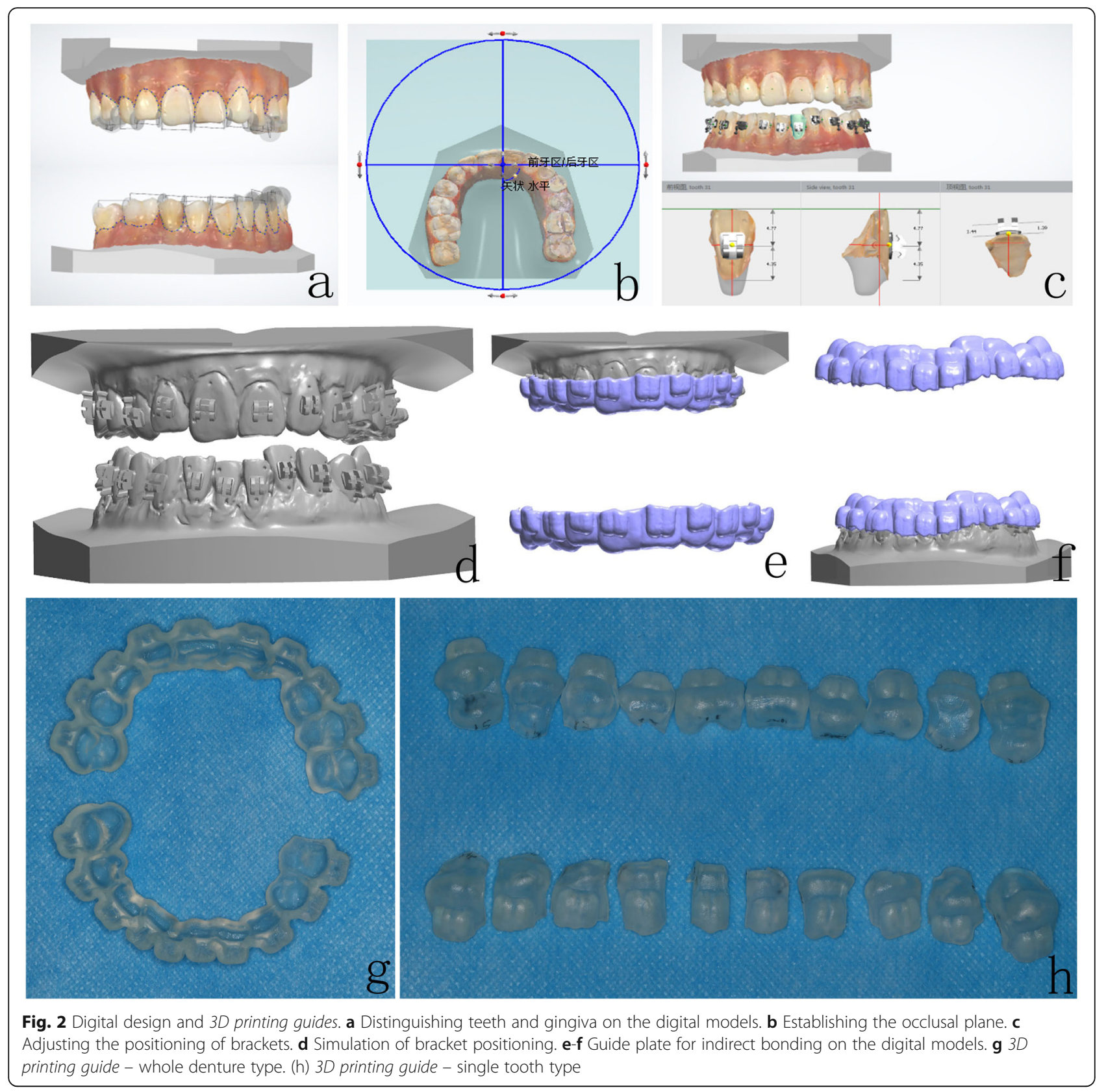

\section{Production of double-layer guide plates and indirect bonding procedure}

A precise impression of the working models with marking points was obtained using silicone-based impression materials (Fig. 4a-b). After 24-h crystallization at the room temperature, plaster casts were duplicated from the silicone molds (Fig. 4c-d). A thin layer of separation agents was applied to the cast tooth surfaces; then, the brackets were positioned and adhered on the crowns using $3 \mathrm{M}$ Transbond $^{\text {Tm }}$ XT light-curable adhesives and light-cured for $5 \mathrm{~min}$ (Fig. 5a-f). Double-layer guide plates were manufactured by Erkoform-3D Thermoformer with a $1 \mathrm{~mm}$ inner layer (soft film) and $0.6 \mathrm{~mm}$ or $0.8 \mathrm{~mm}$ outer layer (hard film). Lastly, we trimmed the excess materials of the inner layer to $2 \mathrm{~mm}$ above the crowns and the outer layer until covering $2 / 3$ of the brackets (Fig. $5 \mathrm{~g}-\mathrm{h}$ ).

Before the indirect bonding procedure, we polished the tooth surfaces, etched buccal/labial surfaces of the crowns for $15 \mathrm{~s}$, and isolated the area with cotton rolls and dried $\mathrm{xx}$ thoroughly. $3 \mathrm{M}$ Sondhi ${ }^{\mathrm{Tm}}$ Rapid-Set Indirect Bonding Adhesive was used for the indirect bonding procedure. The brackets were positioned in the double-layer guide plates $(0.6 \mathrm{~mm}$ or $0.8 \mathrm{~mm}$ outer layer). Solution A was applied to the tooth 

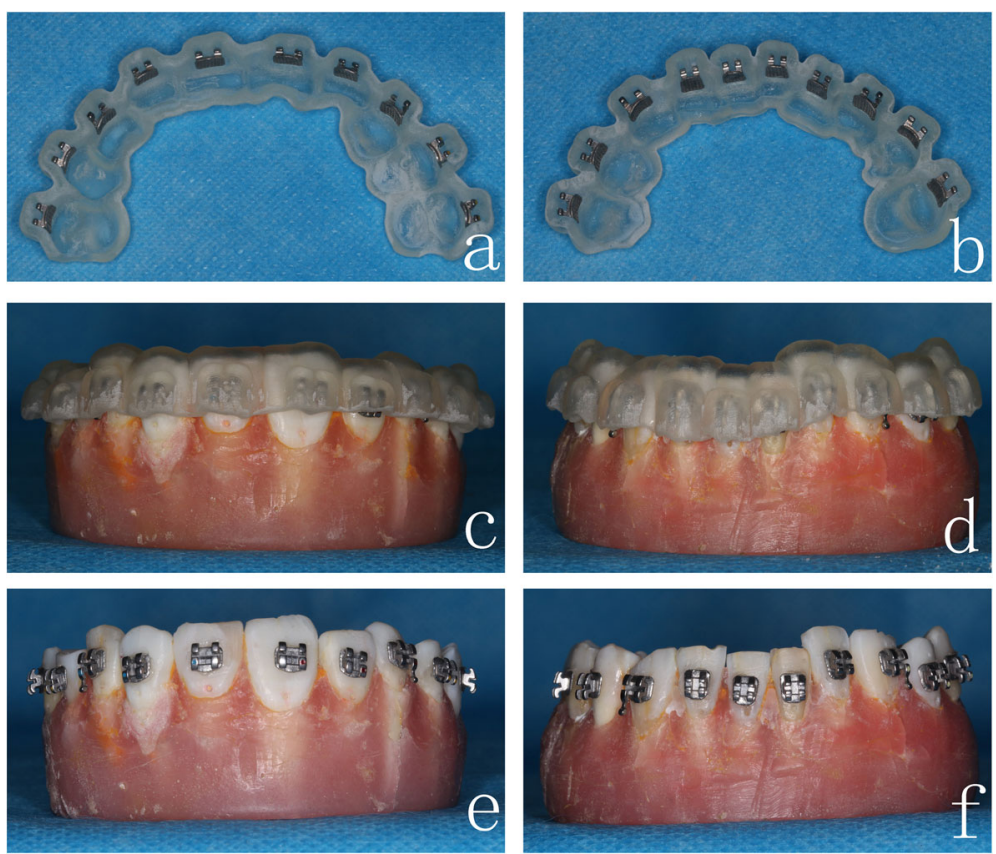

Fig. 3 3D printing guides and indirect bonding procedure. 3D printing guide of the a maxillary and $\mathbf{b}$ mandibular dentitions. 3D printing guides are placed on the $\mathbf{c}$ maxillary and $\mathbf{d}$ mandibular study models. Completion of bracket positioning on the e maxillary and $\mathbf{f}$ mandibular study models

surfaces, and solution B was applied to the base of brackets. The double-layer guide plates were then placed on the study models (Fig. 6a-f). After fixation for $2 \mathrm{~min}$, the outer hard layer was taken off, followed by the inner soft layer. The excess adhesives around the brackets were then removed carefully (Fig. 6g-l).

\section{Measurements}

In the $3 D$ printing guide group, Mimics software was applied to measure the distance between the bracket positions and marking points on the crowns in the digital models. Three measurements were completed and recorded for both designs (i.e., whole denture type and single tooth type). In the double-layer guide plate group,
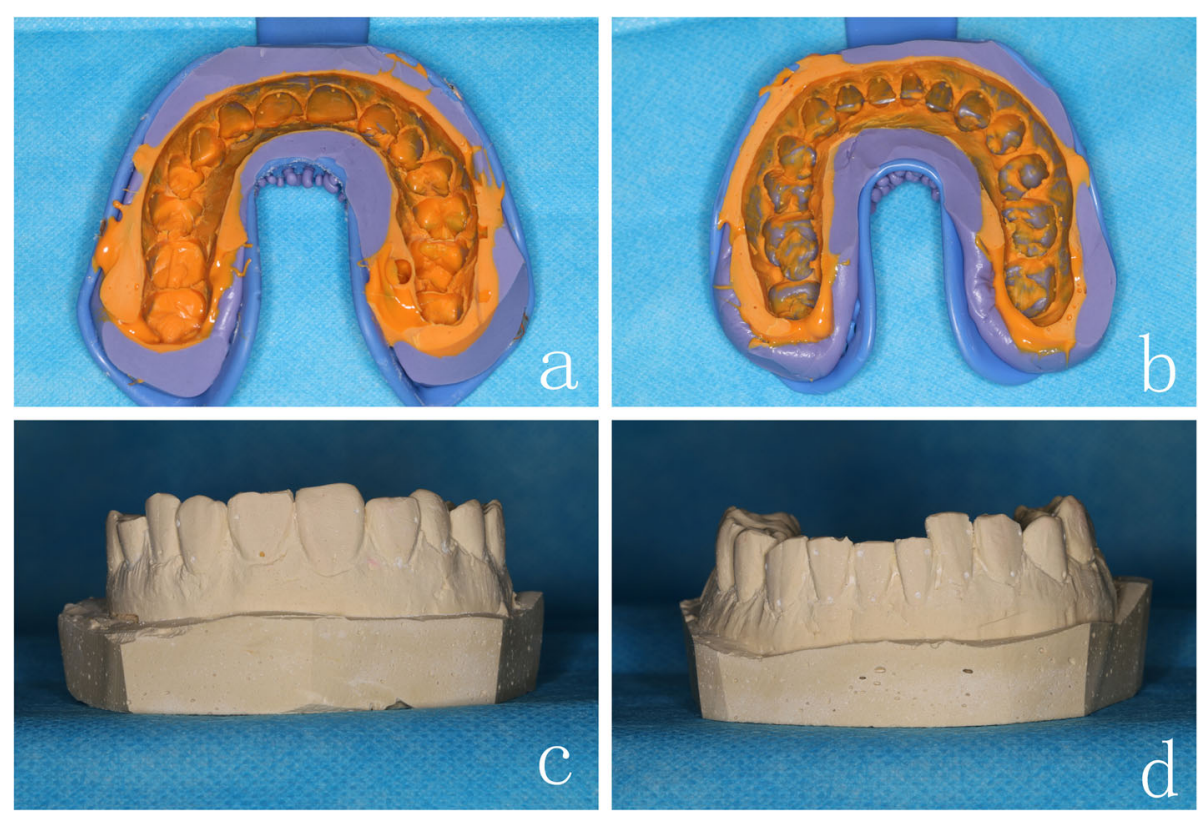

Fig. 4 The impression of a maxillary and $\mathbf{b}$ mandibular dentitions, and the plaster casts of $\mathbf{c}$ maxillary and $\mathbf{d}$ mandibular dentitions 

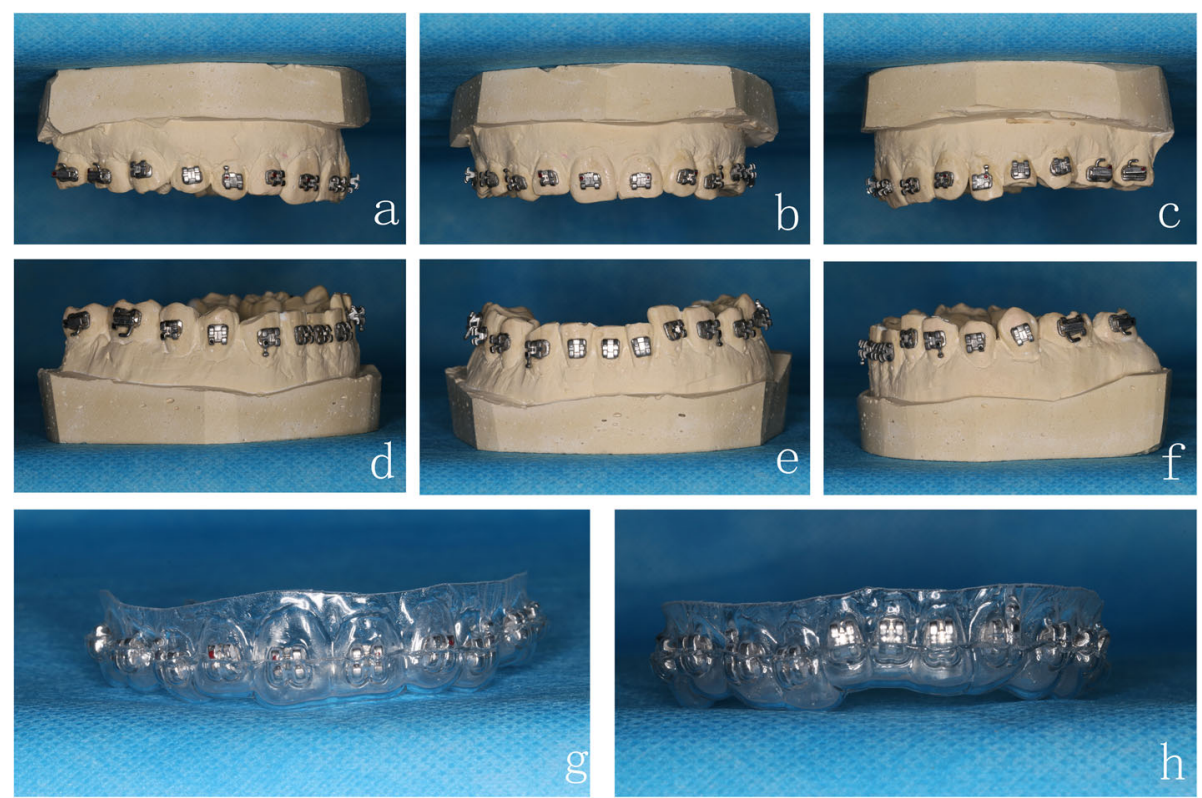

Fig. $\mathbf{5}$ Bracket positioning on the plaster casts and production of double-layer guide plates. Brackes positioning on the a-c maxillary and $\mathbf{d}$-f mandibular dentitions. Double-layer guide plate of the $\mathbf{g}$ maxillary and $\mathbf{h}$ mandibular dentitions
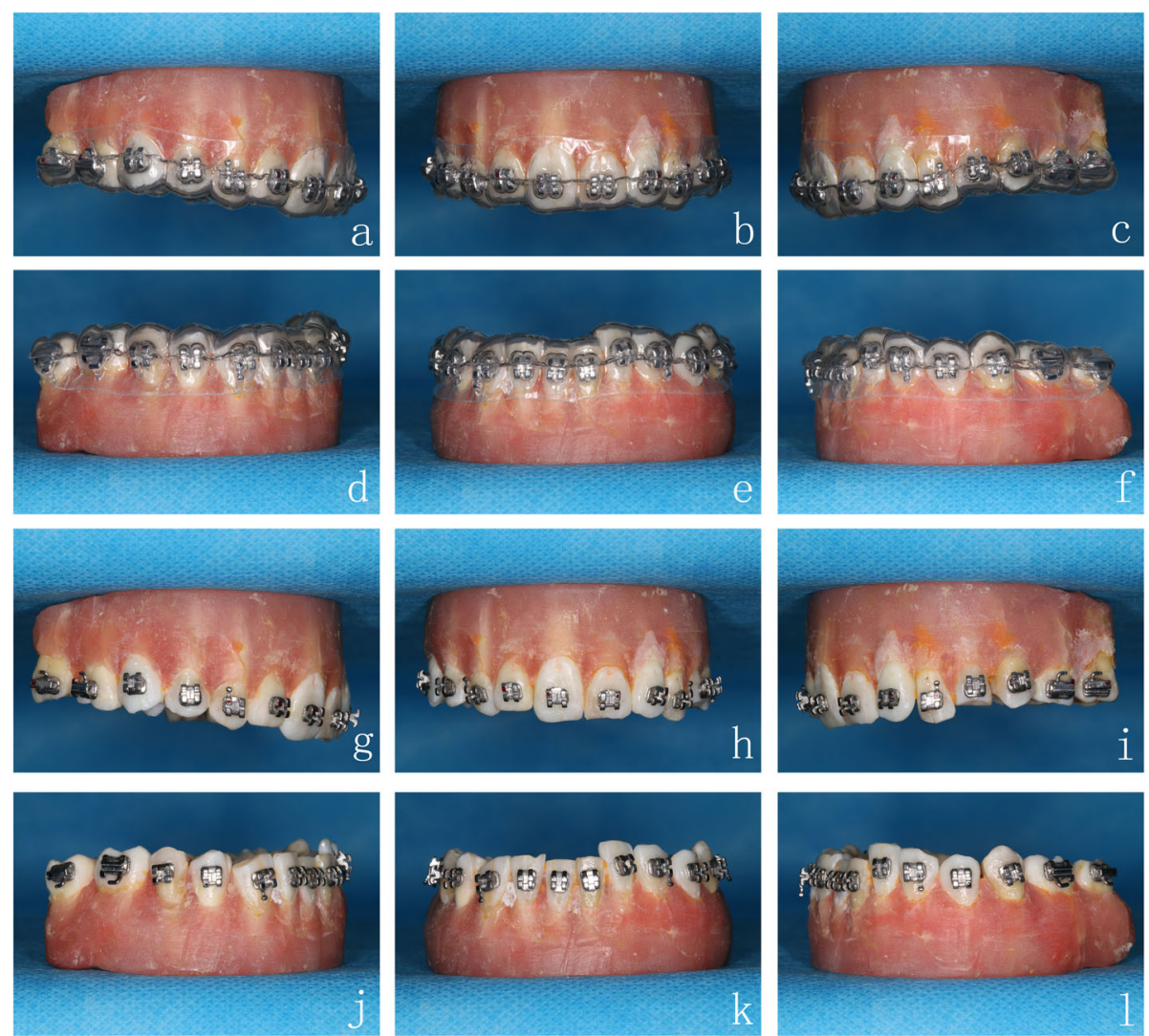

Fig. 6 Double-layer guide plates and indirect bonding procedure. Double-layer guide plates are placed on the a-c maxillary and $\mathbf{d}$-f mandibular study models. Completion of bracket positioning on the $\mathbf{g}$-i maxillary and $\mathbf{j}-\mathbf{I}$ mandibular study models 


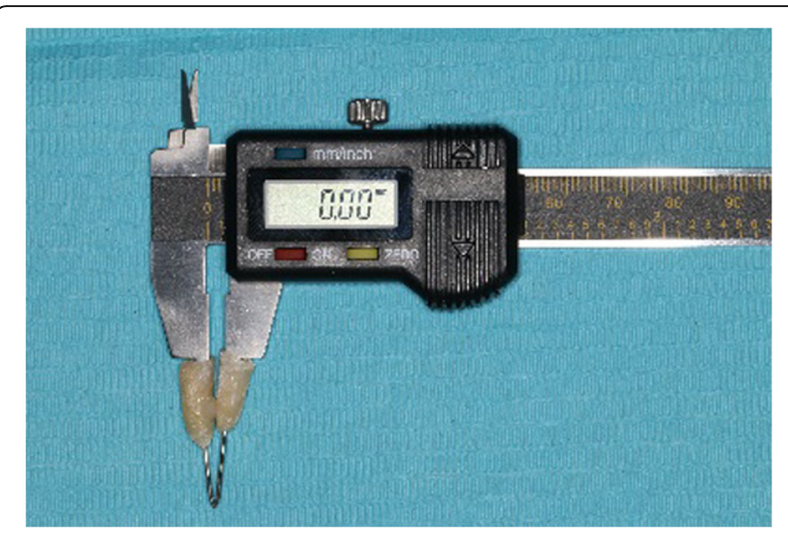

Fig. 7 Electronic caliper

electronic calipers (Fig. 7) were used to measure the distance $\left(\mathrm{d}_{\mathrm{A} 1}, \mathrm{~d}_{\mathrm{B} 1}, \mathrm{~d}_{\mathrm{B} 2}\right.$, and $\left.\mathrm{d}_{\mathrm{C} 2}\right)$ between the bracket positions and marking points on the crowns in the study models (Fig. 8). Three measurements were completed and recorded for both designs (i.e., $0.6 \mathrm{~mm}$ outer layer and $0.8 \mathrm{~mm}$ outer layer).

\section{Statistical analysis}

Statistical analyses were conducted using SPSS 20.0 software. The accuracy of indirect bonding between $3 D$ printing guide and double-layer guide plate was compared using the paired $t$-test. $P<0.05$ indicated statistical significance.

\section{Results}

As shown in Table 1, in the $3 D$ printing guide group, there was no statistical significance in bracket positioning accuracy between the whole denture type and single tooth type $(p=0.078)$. In the double-layer guide plate group, when bracket positioning accuracy was compared using different thicknesses of the outer layer $(0.6 \mathrm{~mm}$ vs.

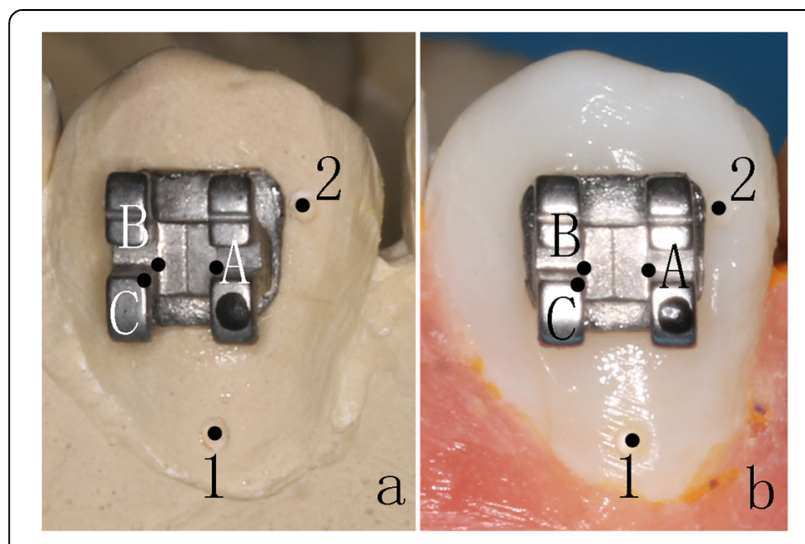

Fig. 8 The marking points on the plaster cast and study model
Table 1 The comparison of different designs in the 3D printing guide group

\begin{tabular}{lccc}
\hline & Single tooth type & Whole denture type & $p$ \\
\hline Distance $(x \pm s, \mathrm{~mm})$ & $0.22 \pm 0.05$ & $0.21 \pm 0.05$ & 0.078 \\
\hline$p<0.05$ & & &
\end{tabular}

$0.8 \mathrm{~mm}$ ), the $0.6 \mathrm{~mm}$ type demonstrated better results $(p=0.036)$, as shown in Table 2.

We then further compared the accuracy of indirect bonding between $3 D$ printing guides (whole denture type) and double-layer guide plates $(0.6 \mathrm{~mm})$, the results were comparable between two groups $(P=0.069)$ (Table 3). However, indirect bonding using double-layer guide plates $(0.6 \mathrm{~mm})$ cost less chair-side time than the $3 D$ printing guides group.

\section{Discussion}

Accurate positioning and bonding of brackets impact on the outcome of orthodontic treatments [1]. In comparison with direct placement of brackets, the indirect bonding procedure significantly decreases chair-side time and improves patient comfort [8]. Indirect bonding was reproducibly demonstrated to be more accurate than direct bonding on bracket positioning with less torque error and rotation deviation $[9,10]$. Furthermore, indirect bonding reduces plaque accumulation and decalcifies white spots around the orthodontic brackets [11]. Though indirect bonding technique becomes popular in orthodontic treatment, there are still some disadvantages, such as timeconsuming and technique-sensitive laboratory procedures and additional expenses on materials [7]. There are many types of transfer trays for indirect bonding, such as double-layer guide plates and $3 D$ printing guides. Overall, double-layer guide plates exhibit shorter time for both fabrication and clinical bonding.

The concept of double-layer guide plates for indirect bonding was initially proposed in 1990s; thermoplastic and silicone-based materials are commonly used to produce transparent transfer trays [12, 13]. Double-layer guide plates are made on super-hard plaster casts with the aid of thermoformers [7]. Both silicone-based impression material and super-hard plaster exhibit optimal stability, with a deformation rate of $0.05 \%$ in the former and $0.1 \%$ in the latter [7]. Typically, the outer layer is rigid to ensure the stability of bracket positioning, and the inner layer is soft to ease removal after bracket transfer. In our preliminary studies, we tested different thicknesses of outer and inner layers. The results revealed that $1 \mathrm{~mm}$ soft film as

Table 2 The comparison of different designs in the double-layer guide plate group

\begin{tabular}{lccc}
\hline & $0.6 \mathrm{~mm}$ outer layer & $0.8 \mathrm{~mm}$ outer layer & $p$ \\
\hline Distance $(x \pm s, \mathrm{~mm})$ & $0.22 \pm 0.08^{*}$ & $0.24 \pm 0.08$ & 0.036 \\
\hline${ }^{*} p<0.05$ & & &
\end{tabular}


Table 3 The comparison of bracket positioning accuracy between 3D printing guide and double-layer guide plate

\begin{tabular}{lccc}
\hline & $\begin{array}{c}\text { Double-layer guide } \\
\text { plate }(0.6 \mathrm{~mm})\end{array}$ & $\begin{array}{l}\text { 3D printing guide } \\
\text { (Whole denture type) }\end{array}$ & $p$ \\
\hline Distance $(x \pm s, \mathrm{~mm})$ & $0.22 \pm 0.08$ & $0.21 \pm 0.05$ & 0.069 \\
\hline$p<0.05$ & & &
\end{tabular}

the inner layer with either $0.6 \mathrm{~mm}$ or $0.8 \mathrm{~mm}$ hard film as the outer layer allowed the best accuracy and stability. Therefore, in the following experiments, we adopted the setting to manufacture double-layer guide plates.

3D printing is an additive manufacturing (AM) and rapid prototyping (RP) technology [14]. The first 3D printing machine was introduced in 1986 and incorporated with stereolithography appearance (SLA), selective laser sintering (SLS), fused deposition modeling (FDM), and laminated object manufacturing (LOM) [14]. The 3D printing technology is highly flexible and customizable, which allows timely production of individualized subjects [14]. Meanwhile, it has a high resolution for detailed designs ( $\sim 0.01 \mathrm{~mm}$ horizontally and $\sim 0.2 \mathrm{~mm}$ vertically) [14]. With all these features, 3D printing is now widely applied in dentistry, in particular, to generate customized brackets, orthodontics models, and guide plates [14]. In the current study, we used 3D printing technology to design two indirect bonding guide plates, whole denture type and single tooth type.

Multiple methodologies have been applied to evaluate the transfer accuracy of indirect bonding. Digital photography or $\mathrm{CBCT}$ was performed to capture the images of study casts and compare bracket positioning before and after the transfer $[10,15,16]$. Based on the study design by Castilla et al., the linear distance (mesiodistal, buccolingual, vertical) and angular differences between the intended and actual bracket position were measured and recorded for further analyses $[16,17]$. In the current study, we aimed to investigate bracket placement accuracy with different indirect bonding guide plates, namely double-layer guide plates and $3 D$ printing guides. According to our preliminary studies for the double-layer guide plates and $3 D$ printing guides, the positioning discrepancy after the brackets transfer was $-0.022 \mathrm{~mm} \pm 0.089 \mathrm{~mm}$ and $-0.025 \mathrm{~mm} \pm 0.077 \mathrm{~mm}$, respectively. With the setting of $\alpha=0.05, Z_{0.05 / 2}=1.96$, and $\beta=0.20, Z_{0.20}$ is 0.842 . To ensure $95 \%$ confidence interval and power of 0.8 , we will need at least 128 (double-layer guide plates) and 75 teeth (3D printing guides) for comparison $\left(\mathrm{N}=\left[\frac{\left(Z_{\alpha}+Z_{\beta}\right) \sigma_{d}}{\delta}\right]^{2}\right)$. To eliminate potential confounding factors, we collected 140 teeth and arranged them into five pairs of full dentition that exhibited mild malocclusion. We measured the distance between brackets and marking points on the tooth surfaces before and after bracket transfer. Paired $t$-test was performed to compare transfer accuracy between $3 D$ printing guides and double-layer guide plates.
Our study demonstrated a significant difference between the $0.6 \mathrm{~mm}$ group and $0.8 \mathrm{~mm}$ group when using double-layer guide plates $(P<0.05)$, with the $0.6 \mathrm{~mm}$ group exhibiting a better transfer accuracy. Double-layer guide plates were produced by a thermoformer, and the thickness of films is proportional to the processing time. When double-layer guide plates are manufactured, both the impression material and plaster have some elasticity, which may lead to subtle transformation and clinical inaccuracy. Therefore, the long processing time negatively affects the transfer accuracy of guide plates. On the other hand, the design of $3 D$ printing guides was completed digitally and printed directly with no material deformation involved in the entire process. However, the accuracy of $3 D$ printing guides is greatly affected by the collection of digital images and file transformation. With minimized human errors, both whole denture type and single tooth type of $3 D$ printing guides exhibited optimal bracket transfer accuracy. The single tooth type needs to be bonded individually, while the whole denture type can be delivered more efficiently. With the aid of computer software, $3 D$ printing guides has numerous advantages. For example, CBCT images of roots and jawbone morphology can be incorporated into the treatment plan to avoid adverse events of orthodontic treatments (e.g., unparalleled roots and dehiscence). This will enhance the predictability and accuracy of the clinical outcome.

Interestingly, the transfer accuracy of indirect bonding is comparable when using $3 D$ printing guides (whole denture type) and double-layer guide plates $(0.6 \mathrm{~mm})$. Though no statistical significance was shown in our data, the overall discrepancy before and after bracket transfer was lower in the $3 D$ printing guides group. This finding might be due to our in vitro study models with only mild malocclusion. Further in vivo studies in more severe clinical cases, such as malocclusion with torsion/tilting/overlapping, will be essential to investigate the efficacy and generalizability of $3 D$ printing guides and double-layer guide plates.

\section{Conclusion}

The accurate positioning and bonding of brackets set the foundation for effective orthodontic treatments [1]. The indirect bonding technique using either double-layer guide plate or $3 D$ printing guide sufficiently increases the accuracy of bracket placement. Double-layer guide plate is a conventional strategy with shorter fabrication time. On the other hand, $3 D$ printing guide combined with digital imaging software demonstrates many advantages and is gaining more and more popularity in orthodontic treatments. In the current study, we utilized five pairs of dentition with mild malocclusion and demonstrated that both guide plates have similar accuracy for bracket transfer. In particular, double-layer guide plates with the $0.6 \mathrm{~mm}$ outer layer exhibited better bracket transfer accuracy than 
the $0.8 \mathrm{~mm}$ group. The study was done in vitro without considering the impact of the tongue, the buccal/labial mucosa, and saliva. Therefore, it is essential to have a well-designed in vivo study to address other physiological factors.

\section{Abbreviations}

3D: Three-dimensional; SLA: Stereolithography apparatus; SLS: Selective laser sintering; FDM: Fused deposition modeling; LOM: Laminated object manufacturing; AM: Additive manufacturing; RP: Rapid prototyping; CBCT: Cone beam computed tomography

\section{Acknowledgements}

None.

\section{Authors' contributions}

$C Y, Y Z$ and $Y L$ have made substantial contributions to conception and design of the study, and written the manuscript; DX, TS and CL searched literature, extracted data from the collected literature and analyzed the data; $C Y$ and $Y Z$ revised the manuscript. All authors approved the final version of the manuscript. All persons named as authors warrant that they have reviewed and approved the manuscript prior to submission.

\section{Funding}

This research was supported by Capital Clinical Application Research and Results Promotion Project (No. Z171100001017154), General Hospital Transformation Project (2017TM-030) and General Hospital Clinical Research Supporting Fund (2018FC-304 M-TSYS-05). The funding bodies were not involved in the design of the study, data collection, analysis, or interpretation of the data and writing the manuscript.

\section{Availability of data and materials}

The datasets generated and analyzed during the current study are available from the corresponding author on reasonable request.

\section{Ethics approval and consent to participate}

Ethical approval was obtained from the Institutional Review Boards of the Fourth Medical Center, Chinese PLA General Hospital. Written informed and oral consent was obtained from the study participants.

\section{Consent for publication}

Not applicable.

\section{Competing interests}

There is no competing interest.

Received: 16 December 2019 Accepted: 12 April 2020

Published online: 28 April 2020

\section{References}

1. Gange P. The evolution of bonding in orthodontics. Am J Orthod Dentofac Orthop. 2015;147(4 Suppl):S56-63.

2. Li Y, Mei L, Wei J, Yan X, Zhang X, Zheng W. Effectiveness, efficiency and adverse effects of using direct or indirect bonding technique in orthodontic patients: a systematic review and meta-analysis. BMC Oral Health. 2019;19(1):137.

3. Silverman E, Cohen M, Gianelly AA, Dietz VS. A universal direct bonding system for both metal and plastic brackets. Am J Orthod. 1972:62(3):236-44

4. Hodge TM, Dhopatkar AA, Rock WP, Spary DJ. A randomized clinical trial comparing the accuracy of direct versus indirect bracket placement. J Orthod. 2004;31(2):132-7.

5. Kwon SY, Kim Y, Ahn HW, Kim KB, Chung KR, Kim Sunny SH. Computeraided designing and manufacturing of lingual fixed orthodontic appliance using 2D/3D registration software and rapid prototyping. Int J Dent. 2014; 2014:164164.

6. Wendl B, Droschl H, Muchitsch P. Indirect bonding--a new transfer method. Eur J Orthod. 2008:30(1):100-7.

7. Nojima LI, Araujo AS, Alves JM. Indirect orthodontic bonding--a modified technique for improved efficiency and precision. Dental Press J Orthod. 2015;20(3):109-17
8. Joiner M. In-house precision bracket placement with the indirect bonding technique. Am J Orthod Dentofac Orthop. 2010;137(6):850-4.

9. Shpack N, Geron S, Floris I, Davidovitch M, Brosh T, Vardimon AD. Bracket placement in lingual vs labial systems and direct vs indirect bonding. Angle Orthod. 2007;77(3):509-17.

10. Nichols DA, Gardner G, Carballeyra AD. Reproducibility of bracket positioning in the indirect bonding technique. Am J Orthod Dentofac Orthop. 2013;144(5):770-6.

11. Dalessandri D, Dalessandri M, Bonetti S, Visconti L, Paganelli C. Effectiveness of an indirect bonding technique in reducing plaque accumulation around braces. Angle Orthod. 2013:82(2):313-8.

12. Hickham JH. Predictable indirect bonding. J Clin Orthod. 1993;27(4):215-7.

13. Sondhi A. Efficient and effective indirect bonding. Am J Orthod Dentofac Orthop. 1999;115(4):352-9.

14. Groth C, Kravitz ND, Jones PE, Graham JW, Redmond WR. Three-dimensional printing technology. J Clin Orthod. 2014;48(8):475-85.

15. Grunheid T, Lee MS, Larson BE. Transfer accuracy of vinyl polysiloxane trays for indirect bonding. Angle Orthod. 2016;86(3):468-74.

16. Castilla AE, Crowe JJ, Moses JR, Wang M, Ferracane JL, Covell DA Jr. Measurement and comparison of bracket transfer accuracy of five indirect bonding techniques. Angle Orthod. 2014;84(4):607-14.

17. Kim J, Chun YS, Kim M. Accuracy of bracket positions with a CAD/CAM indirect bonding system in posterior teeth with different cusp heights. Am J Orthod Dentofac Orthop. 2018;153(2):298-307.

\section{Publisher's Note}

Springer Nature remains neutral with regard to jurisdictional claims in published maps and institutional affiliations.
Ready to submit your research? Choose BMC and benefit from:

- fast, convenient online submission

- thorough peer review by experienced researchers in your field

- rapid publication on acceptance

- support for research data, including large and complex data types

- gold Open Access which fosters wider collaboration and increased citations

- maximum visibility for your research: over $100 \mathrm{M}$ website views per year

At BMC, research is always in progress.

Learn more biomedcentral.com/submissions 